\title{
Self-adapting Classification System for Swallow Intention Detection in Dyspha- gia Therapy
}

Benjamin Riebold, Control Systems Group - TU Berlin, Berlin, Germany, riebold@ control.tu-berlin.de Thomas Schauer, Control Systems Group - TU Berlin, Berlin, Germany, schauer@ control.tu-berlin.de Holger Nahrsteadt, Hasomed GmbH, Magdeburg, Germany, holger.nahrstaedt@ hasomed.de

Rainer Seidl, Unfallkrankenhaus Berlin, Berlin, Germany, rainer.seidl@ukb.de

In dysphagia the ability of elevating the larynx and hyoid is usually impaired. Electromyography (EMG) and Bioimpedance (BI) measurements at the neck can be used to detect and to assess swallowing. The height and speed of larynx elevation can be assessed by evaluating the BI during a swallow. An online detection of swallow intention is required to trigger a supportive functional electrical stimulation (FES) of swallowing related muscles and to enable biofeedback. The success of the stimulation, the biofeedback therapy and the acceptance by the patient strongly depends on the precise detection of swallow intention. We have previously introduced a classification algorithm based on a random forest classifier to trigger FES, that is successful in healthy subjects. Still, difficulties appear in the utilization on some patients. The reason for this can be found in a strongly varying residual swallow activity. Thus, an adaption procedure is needed, that can be easily applied by the medical staff. In this paper we introduce an algorithm that is capable to find an optimal classifier for a patient in terms of sensitivity. The adaption algorithm uses a small number of recorded swallow intentions of a patient, at the beginning of a therapy session, to evaluate a set of different classifiers and to pick the most suitable for the treatment. The evaluation is done using data from 41 patients, to show that the sensitivity of the classification increased from 29.5 to 46.4 percent in average. Finally, we studied the effect of this adaptive classifier in triggered stimulation therapy in a single dysphagia patient. Swallowing performance was measurements during one week of therapy consisting of three therapy sessions. An improvement of 30 and 49 percent in larynx elevation and speed could be observed, respectively. 


\section{Testing of 3D-printed prosthetic sockets: design of a test device with different mounting adaptors.}

Peter Seufert*, Bettina Westebbe*, Marc Kraft*, *Department of Medical Engineering, Technische Universität Berlin, Germany, peter.seufert@tu-berlin.de

Due to the demand of individual prosthetic sockets, the research project "ADDcarbori" (funded by the IB-Berlin) explores the engineering of sockets for lower limb amputees with additive manufacturing methods. These new methods allow to produce sockets with individual shapes and optimized weight in a short period of time. There are two needs for a test device for such sockets. First it is crucial to gather knowledge concerning the mechanical properties in comparison to traditional crafted sockets and second, testing is needed to identify an optimized design in relation to the occurring loads under realistic conditions. To meet these two requirements, a test device is needed which can apply forces typically occurring in the human gait. As a standardized test method for prosthetic socket testing does not exist, a test device was designed according to test methods and loads of the DIN EIN ISO 10328 and DIN EN ISO 22675. Its main actuator is a magnetic linear drive system which can apply loads up to $2 \mathrm{kN}$ under dynamic conditions. The drive is mounted on a rack, the socket is positioned nearby the actuator and tested with different pulsatile applied loads which represents the loads during gait. To couple the sockets with the machine, several adaptors were designed and tested. For example, one adapter is designed to clamp the socket from the inside, while another is suitable for applying loads on the load bearing areas of the brim of the socket. So the device is able to test under different conditions depending on the mounting adaptors. It allows to identify critical structures of the socket design as well as the overall strength in comparison to other sockets or socket designs. 


\section{Wireless retina implant with large visual field}

Thomas Velten, Fraunhofer IBMT, 66280 Sulzbach, Germany, thomas.velten@ibmt.fraunhofer.de Thorsten Knoll, Fraunhofer IBMT, 66280 Sulzbach, Germany, thorsten.knoll@ibmt.fraunhofer.de Frank Stracke, Fraunhofer IBMT, 66280 Sulzbach, Germany, frank.stracke@ibmt.fraunhofer.de Ronan Le Harzic, Fraunhofer IBMT, 66280 Sulzbach, Germany, ronan.le.harzic@ibmt.fraunhofer.de Tino Jäger, PREMA Semiconductor GmbH, 55129 Mainz, Germany, tino.jaeger@prema.com Michael Rammensee, PREMA Semiconductor GmbH, 55129 Mainz, Germany, michael.rammensee@prema.com Oliver Kurz, PREMA Semiconductor GmbH, 55129 Mainz, Germany, oliver.kurz@prema.com Stephan Klesy, PREMA Semiconductor GmbH, 55129 Mainz, Germany, stephan.klesy@prema.com Kai Januschowski, Augenklinik Sulzbach, 66280 Sulzbach, Germany, kai.januschowski@med.uni-tuebingen.de Loic Sermeus, Augenklinik Sulzbach,66280 Sulzbach, Germany, loicsermeus@gmail.com Peter Szurman, Augenklinik Sulzbach, 66280 Sulzbach, Germany, sek-augen@kksulzbach.de

We present the concept of a novel epiretinal prosthesis, consisting of a foil-based, miniaturized electronic system with wireless optical energy and signal transmission and integrated electrostimulation. With this completely wireless solution, there are no wires leading to or into the eye. The pixel arrays, realized on a flexible foil for the first time, adapt to the curvature of the eye. The aim is achieving a wide-angle projection to create a large visual field. The implant consists of a mosaic-like array of thinned silicon-based photodiodes combined with a polyimide foil substrate. Thin-film stimulation electrodes on the foil substrate are electrically connected to the photodiodes. Projection glasses are used for data and energy transmission. They capture the image with an integrated CMOS camera and transmit it wirelessly and optically to the wide field implant via a diode laser and a scanner device. By amplifying the received image by laser projection, this approach achieves a high light intensity per pixel and permits simultaneous optical transmission of the image information and the energy required for electrostimulation.

The proposed implantable neurostimulator is highly flexible and closely adapts to the contour of the inner layers of the retina. Due to its foil structure, the implant fixes itself via the transretinal suction effect and does not require any special fixation. In contrast to all other retinal prostheses, it is not necessary to fix the proposed implant exactly in place. A slight lateral shift plays no role for the topographic control of the ganglion cells to be stimulated.

The final manuscript will describe the concept for the retinal implant as well as first computer simulations of the electrode geometry and results of experimental investigations related to the optimization of the design and thickness of the photodiodes and to the process of integrating the photodiodes with the foil substrate. 


\section{Personalized cochlear implants: A design study on foil-based substrates to adapt to the helical structure of cochlea}

Thomas Stieglitz, Laboratory for Biomedical Microtechnology-IMTEK, BrainLinks-BrainTools, Bernstein Center Freiburg, Albert-Ludwig-University of Freiburg, Freiburg, Germany, e-mail: thomas.stieglitz@imtek.uni-freiburg.de Calogero Gueli, Laboratory for Biomedical Microtechnology-IMTEK, Albert-Ludwig-University of Freiburg, Freiburg, Germany, e-mail: calogero.gueli@imtek.uni-freiburg.de

Rolf B. Salcher, Department of Otolaryngology, Head \& Neck, Hannover Medical School, Hannover, Germany, e-mail: salcher.rolf@mh-hannover.de

The size of the cochlea varies from patient to patient. Current clinical cochlear implants are not yet tailored to the individual cochlea but are offered in certain lengths of electrode arrays. These arrays are tubular shaped with ring-like electrodes arranged in a linear array. If the number of stimulation channels in cochlear implants shall increase for more specific stimulation at higher spatial resolution, it is of utmost importance that current spreading is limited to prevent short circuits of adjacent channels and reduction of specificity due to leakage currents. Close proximity of the electrodes to the target structure is needed to achieve these target specifications.

Flexible polymer foils with embedded micromachined thin-film metallization allow for higher integration densities of electrodes within an array. However, since the electrodes are on one side of the foil only, they do not deliver an electrical field homogeneously in a cylindrical volume but have a preferential field direction perpendicular to the surface. Straight long foils might not ideally adapt to a helical structure like the cochlea.

We investigated models of different cochlea from guinea pigs and humans to derive ranges of dimensions of curvature, length and inclination of the cochlea. Force development in foils based on their geometrical dimensions (thickness, width) and the target structure curvature was calculated. Spiral-like foils were designed to better adapt to the helical structure of the cochlea. Insertion experiments were first done on transparent models of the cochlea to monitor the insertion behaviour as well as the adaptation of the foils to the target walls. Our pilot studies indicate that design of foils substrate with shapes that allow threedimensional adaptation to the cochlea improve placement and orientation. Insertion tools and methods have to be co-developed to these new substrates that are comparable to insertion of precision mechanics manufactured arrays. 


\section{Micromachined transducer for implant-to-implant communication via ultra- sound}

Thomas Velten, Fraunhofer IBMT, 66280 Sulzbach, Germany, thomas.velten@ibmt.fraunhofer.de Thorsten Knoll, Fraunhofer IBMT, 66280 Sulzbach, Germany, thorsten.knoll@ibmt.fraunhofer.de Adam Sossalla, Fraunhofer IBMT, 66280 Sulzbach, Germany, adam.sossalla@ibmt.fraunhofer.de Jorge Oevermann, Fraunhofer IBMT, 66280 Sulzbach, Germany, jorge.oevermann@ibmt.fraunhofer.de Peter Weber, Fraunhofer IBMT, 66280 Sulzbach, Germany, peter-karl.weber@ibmt.fraunhofer.de Frank Tiefensee, Fraunhofer IBMT, 66280 Sulzbach, Germany, frank.tiefensee@ibmt.fraunhofer.de Roman Ruff, Fraunhofer IBMT, 66280 Sulzbach, Germany, roman.ruff@ibmt.fraunhofer.de Andreas Schneider, Fraunhofer IBMT, 66280 Sulzbach, Germany, andreas.schneider-ickert@ibmt.fraunhofer.de Klaus-Peter Hoffmann, Fraunhofer IBMT, 66280 Sulzbach, Germany, Klaus-Peter.Hoffmann@ibmt.fraunhofer.de

Implanted medical devices with multilocular signal acquisition or intervention nowadays use sensors/actuators connected to the implant via a cable. This leads to complex revisions. Desirable are distributed miniaturized implants that can be controlled by wirelessly communicated commands. Future areas of application include gripping neuroprostheses, intestinal pacemakers and scoliosis therapy. We present a capacitive micromachined ultrasonic transducers (cMUT) designed for application in ultrasound-based wireless communication between implants. Typically, a much higher bandwidth can be achieved with cMUTs in comparison with traditional piezoelectric transducers based on ceramic materials. This is particularly advantageous when using ultrasound for data communication. The cMUTs presented here use monocrystalline silicon as material of the vibrating membranes of the individual cells of the transducer array. In contrast to ultrasound arrays for imaging applications, all cells of the array are electrically connected in parallel. Transducer production starts with thermally growing a thin silicon dioxide layer on a doped silicon wafer. Wet chemical patterning of the oxide layer is applied for defining the distance between the silicon wafer and the later membranes in the membrane areas and thus the electrode spacing of the capacitive transducer elements. Subsequently, fusion bonding of an SOI wafer and the removal of the handle wafer and the oxide layer of the SOI wafer take place. The device layer of the SOI wafer forms the membranes of the individual transducer elements. The individual capacitors of the capacitive ultrasound transducer are build by a metal electrode sputter-deposited on each membrane and the highly doped silicon wafer which acts as a common counter electrode.

The cMUT design was optimized by computer simulations according to the finite element method using the softwarepackage PZFlex. The dimensions have been chosen in a way that the natural frequency of the vibrating membranes, which are electrically pre-deflected by a DC voltage of $60 \mathrm{~V}$, is approx. $1 \mathrm{MHz}$.

We will describe the concept of implant-to-implant communication in more depth and will further include a comparison of the computer simulation results with the results of the characterization of the produced cMUTs. 


\section{Modification of joint prosthesis surfaces by ultrashort pulse laser treatment for improved joint lubrication}

Philipp Drescher, Microfluidics, University of Rostock, Rostock, Germany, philipp.drescher@uni-rostock.de Paul Oldorf, SLV Mecklenburg-Vorpommern GmbH, Rostock, oldorf@slv-rostock.de

Tim Dreier, Microfluidics, University of Rostock, Rostock, Germany, tim.dreier@uni-rostock.de Rigo Peters, SLV Mecklenburg-Vorpommern GmbH, Rostock, peters@slv-rostock.de

Hermann Seitz, Microfluidics, University of Rostock, Rostock, Germany, hermann.seitz@uni-rostock.de

Endoprostheses such as hip replacements are subject to wear. Approximately $10 \%$ of these implants undergo revisions within a 15-year lifespan per device. Lubrication of the implanted joint interface plays a key role in the wear process, but its mechanism is challenging to understand. The main issue is the three-body abrasion which leads to a shorter life cycle. In order to improve the life cycle, the surfaces of the articulating components can be modified, for example by pulsed femtosecond-laser structuring. By applying microstructures to the surface of the implant, the synovia fluid between the joint can exhibit an increase in viscosity, leading to better lubrication and therefore lower particle abrasion. The objective of this study was to evaluate different microstructures and the resulting flow properties of a joint fluid substitute.

Various microstructures were investigated in a modified rheometer setup. The modification of the rheometer aims to decrease the gap between both surfaces to $100 \mu \mathrm{m}$ in order to increase the influence of the microstructures on the test fluid. As a test fluid, a synovia substitute was used, consisting of fetal calf serum and adjusted with hyaluronic acid in order to resemble the fluid properties of the synovia. The test samples for micro structuring consist of CoCrMo disks with a diameter of $25 \mathrm{~mm}$ and a height of $6 \mathrm{~mm}$ with a surface roughness of $\mathrm{Ra} \bullet 0.1 \mu \mathrm{m}$, in accordance to the standard DIN ISO 6476.

The results show that an increase in the viscosity of the synovia substitute can be achieved by micro structuring. An increase of viscosity of up to $20 \%$, compared to the unstructured reference, was observed with ring-structures with a diameter of $100 \mu \mathrm{m}$ and a depth of $20 \mu \mathrm{m}$. Further investigations of various microstructures will help determine an optimal pattern for optimal lubrication. 


\section{The Development of an Algorithm to Quantify the Quality of Autonomously Performed Rehabilitation Exercises}

Asaad Sellmann, Department of Rehabilitation and Prevention Engineering, Institute of Applied Medical Engineering, RWTH Aachen University, Aachen, Germany, sellmann@ame.rwth-aachen.de

Jörg Eschweiler, Department of Orthopaedic Surgery, RWTH Aachen University Hospital, Aachen, Germany, joeschweiler@ukaachen.de

Catherine Disselhorst-Klug, Department of Rehabilitation and Prevention Engineering, Institute of Applied Medical Engineering, RWTH Aachen University, Aachen, Germany, disselhorst-klug@ame.rwth-aachen.de

It has been shown that exercise reduces pain and increases function in patients with low back pain. Rehabilitation exercises are typically designed to optimize muscle activation and coordination. Therefore, it is important that patients adhere to instructions especially when performing exercises autonomously. During autonomous exercise, patients are facing a higher risk of injury through missing guidance and control. Thus, methods to monitor the execution of rehabilitation exercises are needed to prevent secondary damage related to incorrect performance.

IMU Sensors, placed at L5, C7 and the back of the head, are used to detect the movement of the back during exercise performance. To generate a test data set, volunteers were asked to perform different exercises in two cases: clinically prescribed exercise (CPE) and typical compensatory movement (TCM). CPEs are optimized to ensure proper form and the TCMs are designed to reflect typical performance mistakes.

From the signals, a set of $\mathrm{N}$ parameters has been identified that focus on the reflection of physiological events. Out of these $\mathrm{N}$ parameters, six parameters were extracted which have the highest capacity to differentiate between CPE and TCM. In classifying the exercise performance the six parameters were normalized and weighted with respect to their discrimination ability. Subsequently a score was formed by summation, which reflects the quality of exercise execution. For score validation, a study with 30 healthy subjects was conducted who performed three different exercises: ProneRocking, Bird-Dog and Rowing. In each case a subject was instructed to execute 3 sets of 6 repetitions for every exercise. The order of cases i.e. CPE and TCM and exercises was randomised for each subject.

The results showed that the score is able to distinguish CPE from TCM exercises. Thus the introduced score can be used to evaluate autonomously performed exercises in the future. 